\title{
Auf „Kortison“-Angst eingehen
}

mmer noch lehnen viele Patienten die Behandlung mit Glukokortikoiden ab, so eine aktuelle Umfrage. Von März

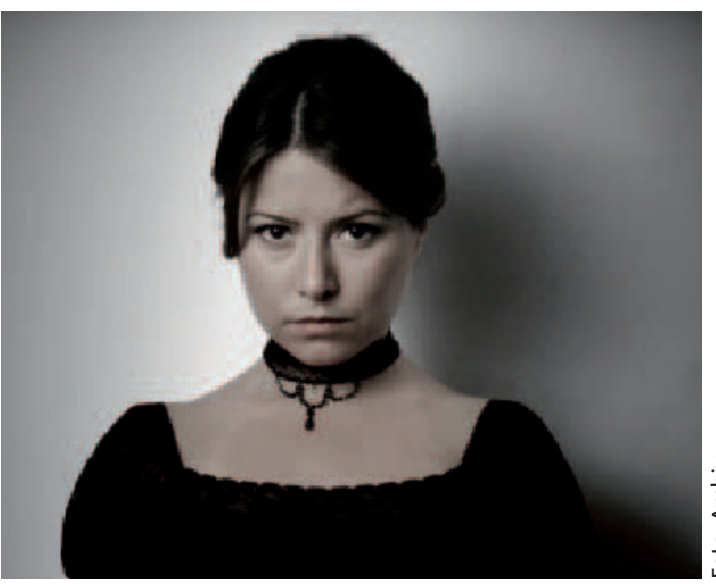

Vorbehalte gegenüber topischen Glukokortikoiden sind immer noch verbreitet.

bis September 2007 wurden 807 Patienten mit allergischer Rhinitis oder atopischer Dermatitis, 1.632 Patienten ohne chronische Krankheiten sowie 453 Ärzte online und in Gruppendiskussionen befragt. Zwei Drittel der Patienten mit allergischer Rhinitis (AR, 64\%) und 43\% der Patienten mit atopischer Dermatitis (AD) haben danach eine Steroidphobie. $82 \%$ der Patienten mit AR und
$87 \%$ der Patienten mit AD glauben, dass Steroide zu Gewichtszunahme, Hautveränderungen und Müdigkeit führen. Dabei machten beide Gruppen keinen Unterschied zwischen systemischen und niedrig dosierten lokal wirkenden Präparaten. Außerdem wussten sie selten etwas über die Entzündungshemmung als wichtigste gewünschte Wirkung des „Kortisons“.

Hauptinformationsquelle in Sachen Behandlung ist nach der Umfrage weiterhin der Arzt. Deshalb sollten behandelnde Ärzte im Gespräch auf die Unsicherheiten und Bedenken der Patienten eingehen. Laut Studie förderte die Erklärung des Arztes, er verschreibe ein ,niedrig dosiertes modernes und nur lokal in der Schleimhaut wirkendes Kortison", signifikant die Bereitschaft, ein solches Präparat anzuwenden.

Wichtig war den Patienten die Information über objektive Studienergebnisse zu Risiken und Nebenwirkungen gefolgt von Vergleichen der Wirksamkeit von Medikamenten mit oder ohne Glukokortikoide. Als Drittes wünschten sie sich Informationen über die Langzeitanwendung.

Nach Informationen von essex pharma, München

\section{Förderpreis ausgeschrieben}

Die Stallergenes $\mathrm{GmbH}$ hat erstmalig einen Förderpreis ausgeschrieben, mit dem jedes Jahr insgesamt fünf praxisnahe Projekte zur Förderung der spezifischen Immuntherapie ausgezeichnet werden sollen. Der mit je 5.000 Euro dotierte Preis soll an Praxen verliehen werden, die Konzepte oder Projekte entwickelt haben, um die Compliance ihrer Patienten im Rahmen der drei bis fünf Jahre dauernden spezifischen Immuntherapie (SIT) zu verbessern. Die Beurteilung und Auswahl erfolgt durch ein unabhängiges interdisziplinäres Expertengremium unter Schirmherrschaft des Deutschen Allergieund Asthmabundes (DAAB). An der Ausschreibung können sich allergologisch tätige Fachärzte aus Klinik und Praxis beteiligen, die eine SIT durchführen.

Nach Informationen von Stallergenes, Kamp-Lintfort.

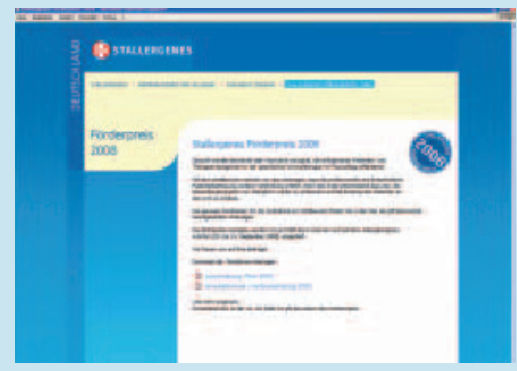

Informationen zum neuen Förderpreis finden sich im Internet unter www.foerderpreis.stallergenes.de

\section{Berliner Gesundheitspreis ausgeschrieben}

G esagt ist nicht getan. Dieses Motto hat der Berliner Gesundheitspreis 2008, den AOK-Bundesverband, $\mathrm{AOK}$, Berlin und Berliner Ärztekammer ausgeschrieben haben. $\mathrm{Er}$ ist mit Preisgeldern von insgesamt 50.000 Euro dotiert.

Hinter dem Slogan steckt das Thema „Adherence“, Therapietreue. Gesucht werden innovative Ideen und Modelle für eine Arzt-PatientenBeziehung, bei der der Patient aktiv und gleichberechtigt in die Therapieplanung und -durchführung einbezogen wird. Ziel ist es dabei, die Therapiemotivation und Theapietreue $\mathrm{zu}$ stärken. Ärzte, Pflegekräfte und The- rapeuten in ambulanten wie stationären Einrichtungen sowie Träger von Forschungsprojekten, die geeignete Konzepte anbieten, können sich an dem Wettbewerb beteiligen. Eine unabhängige Jury aus Vertretern von Politik, Medizin und Wissenschaft entscheidet über die Preisvergabe. Einsendeschluss ist der 30. September 2008. Die Preisverleihung findet im Frühjahr 2009 in Berlin statt. Ausschreibungsunterlagen und Informationen erhalten Sie im Internet unter www.berliner-gesundheitspreis.de

Nach Informationen des AOK-Bundesverbands, Berlin

\section{Neue Rabattpartner}

1 A Pharma hat zum 1. Juni 2008 mit den beiden privaten Krankenversicherern HUK-Coburg Krankenversicherung und PAX Familienfürsorge Krankenversicherung einen Rabattvertrag gemäß $§ 78$ AMG geschlossen.

Der Vertrag umfasst das Gesamtsortiment. Für mehrere hunderttausend Privatversicherte der beiden Krankenversicherer ist damit eine preisgünstige Arzneimittelversorgung sichergestellt.

Die vereinbarten Rabatte werden laut HUK-Coburg vollständig an die Kunden weitergegeben.

Nach Informationen von 1 A Pharma, Oberhaching 